\title{
A Hybrid SSPPs-EBG Filter with Glide Symmetry for 5G Applications
}

\author{
Marzieh SalarRahimi ${ }^{1}$, Guy A.E Vandenbosch ${ }^{2}$ \\ ${ }^{1}$ ESAT-TELEMIC, KU Leuven, Leuven, Belgium, marzieh.salarrahimi@kuleuven.be \\ ${ }^{2}$ ESAT-TELEMIC, KU Leuven, Leuven, Belgium, guy.vandenbosch@kuleuven.be
}

\begin{abstract}
In this paper, we propose a metamaterial-based low-pass band-pass hybrid filter combining the low-pass features of a spoof surface plasmon polaritons transmission line and band-reject features of an edge via mushroom-like glide-symmetric EBG structure. The idea of the proposed hybrid filter is to increase the degrees of freedom to tune the performance. The filter has been designed for 5G applications, covering both the sub $6 \mathrm{GHz}$ and millimeter-wave frequency ranges. A stopband suppression of more than $25 \mathrm{~dB}$ has been achieved.
\end{abstract}

Index Terms-Metamaterial, spoof surface plasmon polaritons, filters, electromagnetic band gap.

\section{INTRODUCTION}

In recent years, periodic structures have widely been engaged into the design of different types of passive microwave devices. The two main features that make these structures distinctive are: a) dispersion characteristics of each individual mode and, b) frequency-selective properties that are set up by band gaps between the modes. Concerning the first feature, for example Spoof Surface Plasmon Polaritons (SSPPs) have the advantage of being able to control their dispersion characteristics and cut-off frequency by tuning the structural parameters. Actually, SSPPs are metasurfaces consisting of periodic metal textures, which can mimic surface plasmons. On the other hand, Electromagnetic Band Gap (EBG) periodically patterned surfaces have the advantage of suppressing all propagating modes in their band gap frequency ranges, fulfilling the second feature. Therefore, using the frequency-selectivity, they can be used to design different filters.

To improve filter performance, the idea of a hybrid filter, by merging different design methods together, was presented. In [1], a microstrip ridged waveguide and EBG surfaces were used to realize a low-loss filter in K-band. In [2], several coupled resonators were integrated into a gap waveguide to make a fifth order Chebyshev filter. In [3], a band-pass filter based on the high-pass features of a half mode substrate integrated waveguide and the use of lowpass features of a double-layer SSPPs were proposed.

In this paper, we propose a low-pass band-pass hybrid filter based on the low-pass features of an SSPPs

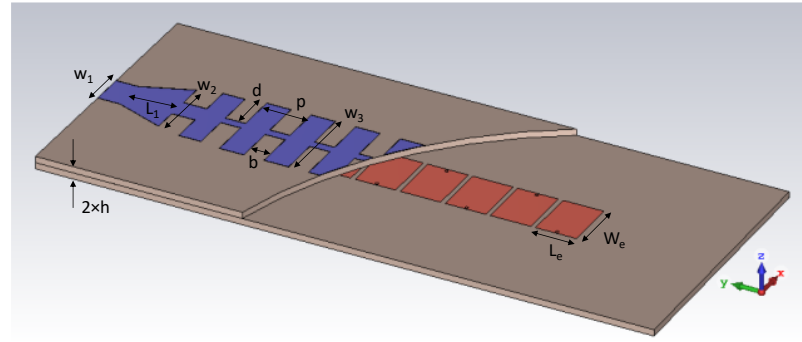

a)

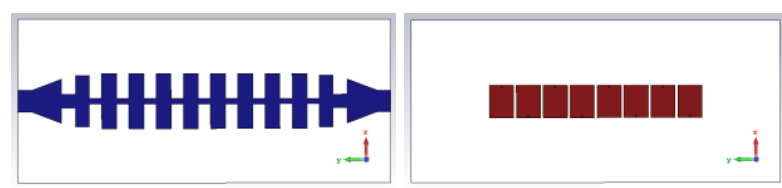

b)

c)

Fig. 1. The proposed filter, a) 3D, b) first layer, c) second layer.

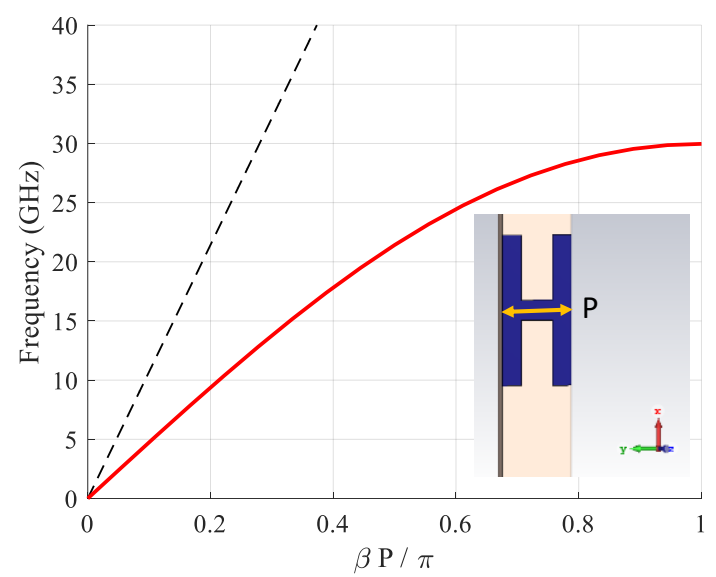

Fig. 2. Unit cell and dispersion diagram of the SSPPs-TL.

transmission line and the band-reject features of a mushroom-like glide-symmetric EBG structure. The idea is to increase the degrees of freedom to tune the performance of the filter. 


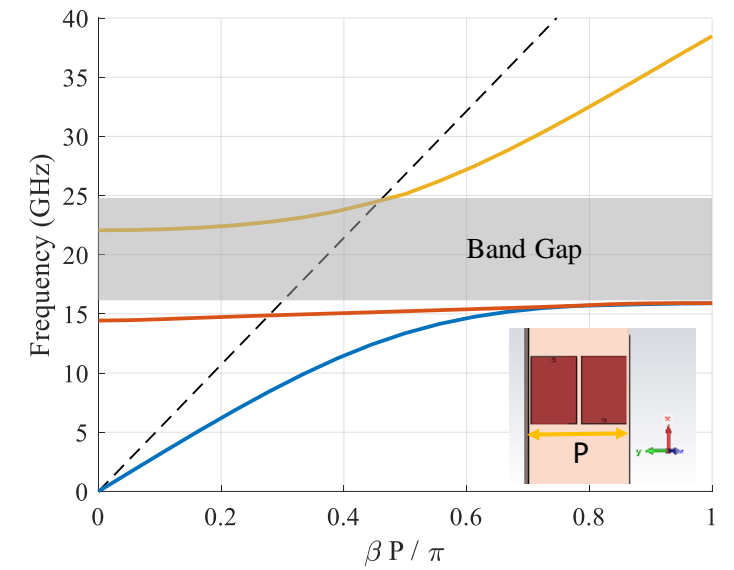

Fig. 3. Unit cell and dispersion diagram of the glide symmetric EBG.

\section{DESIGN AND RESULTS}

Fig. 1 shows the structure of the filter. The goal is to design a filter to cover $5 \mathrm{G}$ frequency bands (sub $6 \mathrm{GHz}$ and $24.25-29.5 \mathrm{GHz}$ ) [5] and reject the signal in other frequency bands with a stopband suppression of more than $25 \mathrm{~dB}$. In this section, the design is described step-by-step.

The first step is to design the SSPPs transmission line, based on the model that was presented in [4]. This transmission line has been selected because it supports slow wave propagation, which makes the filter compact and low loss. Fig. 2 shows the unit cell of the SSPPs transmission line and its dispersion diagram. The parameters have been tuned to get the maximum field confinement over the

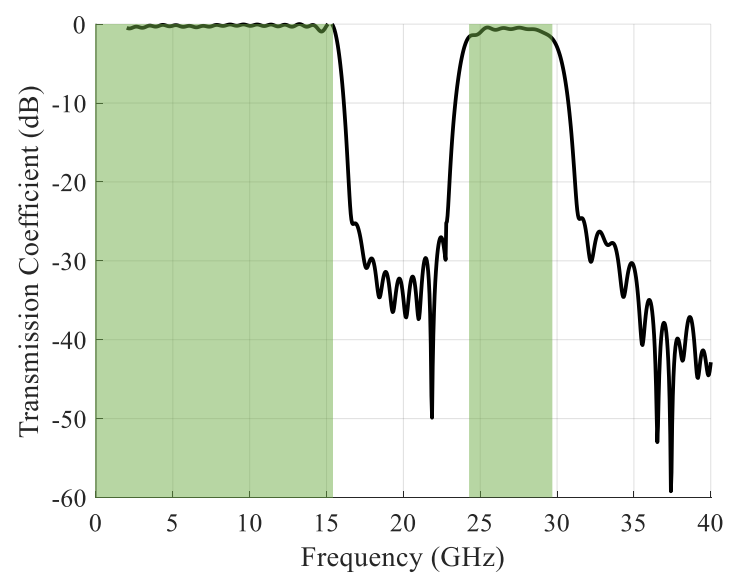

Fig. 5. Transmission coefficient of the proposed low-pass band-pass hybrid filter.

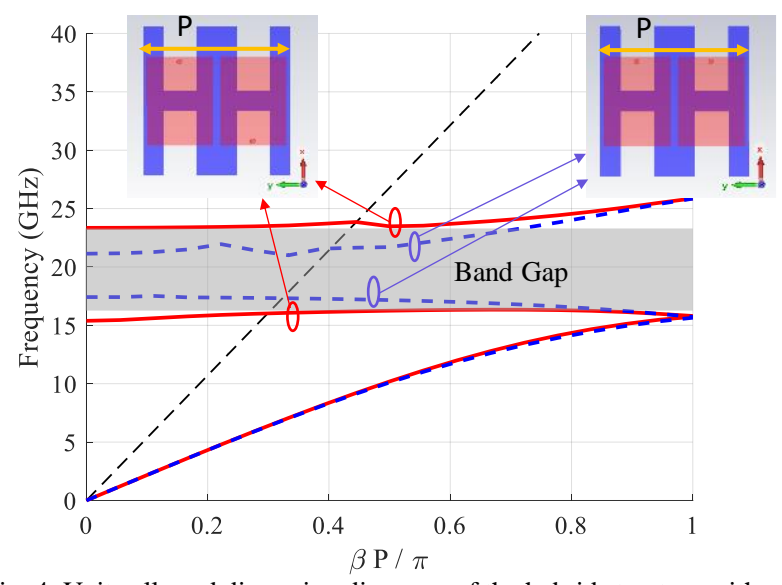

Fig. 4. Unit cells and dispersion diagrams of the hybrid structure with regular and glide symmetric EBG.

transmission line and a cut-off frequency at $30 \mathrm{GHz}$.

The second step is to design the EBG part, which is supposed to create a stopband between both passbands. Therefore, a very wideband EBG is required to cover the whole stopband. To make it simple from a fabrication point of view, the mushroom-like patch based EBG is chosen. Three main techniques are used to make it compact and wideband: a) rectangular patches instead of conventional square patches, b) edge-located via instead of center-located via [6] and, c) applying glide symmetry [7].

Fig. 3 shows the unit cell of the EBG structure, which is periodic in y direction. The dispersion diagram of the EBG is depicted in Fig. 3, with the band gap highlighted. It covers $15.9-24.5 \mathrm{GHz}$.

The next step is to put the SSPPs transmission line on top of the high impedance EBG surface. Two Rogers RT5880 substrates of $0.2 \mathrm{~mm}$ thicknesses contain the transmission line and the mushroom cells. Only one row of EBG cells is sufficient, thanks to the properties of strong field confinement and high local field intensity in the SSPPs transmission line. The parameters of the structure were tuned to adjust the band gap. Fig. 4 shows the dispersion diagram of this combination. To investigate the effect of the applied glide symmetry, dispersion curves of the structure without glide symmetry also are depicted in Fig. 4. Comparing both dispersion diagrams, the applied glide symmetry causes a wider band gap, from $16.3 \mathrm{GHz}$ to 23.5 $\mathrm{GHz}$.

In addition, the filter structure needs a transition to get properly connected to a normal transmission line and be able to be integrated in a larger system. We choose to use microstrip 50-Ohm transmission lines at the input and output of the filter, realized with cost efficient PCB

TABLE I

STRUCTURE PARAMETERS

\begin{tabular}{cccccccccccc}
\hline \hline $\mathrm{W}_{1}$ & $\mathrm{~L}_{1}$ & $\mathrm{~W}_{2}$ & $\mathrm{~d}$ & $\mathrm{p}$ & $\mathrm{B}$ & $\mathrm{W}_{3}$ & $\mathrm{~L}_{\mathrm{e}}$ & $\mathrm{h}$ & \\
\hline 1.1 & 1.5 & 2.26 & 1.27 & 1.4 & 0.63 & 2.8 & 0.2 & 1.68 & 1.26 &
\end{tabular}


technology. The proposed transition is very simple and small in comparison to the conventional transition mode convertor for SSPPs transmission lines [8]. Besides, its performance is adequate in the desired frequency range.

Dimensions of the whole structure, consisting of the SSPPs transmission line, the mushroom-like EBG cells, the transition mode convertor, and the driving microstrip lines are listed in Table I.

Fig. 5 shows the transmission coefficient of the proposed structure. It covers frequencies less than $15.3 \mathrm{GHz}$ and the millimeter-wave $5 \mathrm{G}$ frequency band $(24.25-29.5$ $\mathrm{GHz}$ ).

\section{CONCLUSIONS}

In this conference paper, the low-pass features of a SSPPs transmission line and band-reject features of an edge via mushroom-like glide-symmetric EBG structure are used to make a hybrid filter. The filter has been designed to transfer low frequencies (less than $15.3 \mathrm{GHz}$ ) and the mmwave $5 \mathrm{G}$ band $(24.25-29.5 \mathrm{GHz})$. Therefore, it is called a low-pass band-pass hybrid filter. Preliminary simulation results show a very promising topology with a stopband suppression of more than $25 \mathrm{~dB}$. Implementation of the filter is in progress and detailed measurement results will be presented at the conference

\section{ACKNOWLEDGMENT}

This project has received funding from the European Union's Horizon 2020 research and innovation programme under the Marie Sklodowska-Curie grant agreement No. 721732 .

\section{REFERENCES}

[1] S. Birgermajer, N. Janković, V. Radonić, V. Crnojević-Bengin, and M. Bozzi, "Microstrip-ridge gap waveguide filter based on cavity resonators with mushroom inclusions," IEEE Trans. Microw. Theory Techn., vol. 66, no. 1, pp. 136-146, Jan. 2018.

[2] M. Rezaee and A. U. Zaman, "Realisation of carved and iris groove gap waveguide filter and E-plane diplexer for V-band radio link application," IET Microw., Antennas Propag., vol. 11, no. 15, pp. 2109-2115, Oct. 2017.

[3] L. Zhao, Y. Li, Z.M. Chen, X.H. Liang, J. Wang, X. Shen, and Q. Zhang, "A band-pass filter based on half-mode substrate integrated waveguide and spoof surface plasmon polaritons," Scientific reports, vol. 9, no. 1, pp.1-8, 2019.

[4] A. Kianinejad, Z. N. Chen, and C.-W. Qiu, "Design and modeling of spoof surface plasmon modes-based microwave slow-wave transmission line," IEEE Trans. Microw. Theory Techn., vol. 63, no. 6, pp. 1817-1825, Jun. 2015.

[5] Ercisson AB, "Ericsson microwave outlook. Ercisson AB," Dec. 2018.

[6] E. Rajo-Iglesias, L. Inclan-Sanchez, J. L. Vazquez-Roy, and E. GarciaMuoz, "Size reduction of mushroom-type EBG surfaces by using edgelocated vias," IEEE Microw. Wireless Compon. Lett., vol. 17, no. 9, pp. 670-672, Sep. 2007.

[7] B. A. Mouris, A. Fernandez-Prieto, R. Thobaben, J. Martel, F. Mesa, O. Quevedo-Teruel, "On the Increment of the Bandwidth of Mushroom-typeEBG Structures withGlide Symmetry", submitted to IEEE Transactions on Microwave Theory and Techniques, 2019.

[8] H. F. Ma, X. Shen, Q. Cheng, W. X. Jiang, and T. J. Cui, "Broadband and high-efficiency conversion from guided waves to spoof surface plasmon polaritons," Laser Photon. Rev., vol. 8, no. 1, pp. 146-151, 2014 